\title{
Investigation of the dimensionality of charge transport in organic field effect transistors
}

\author{
Hassan Abdalla, ${ }^{1}$ Simone Fabiano, ${ }^{2}$ and Martijn Kemerink ${ }^{1}$ \\ ${ }^{1}$ Department of Physics, Chemistry and Biology, Linköping University, 58183 Linköping, Sweden \\ ${ }^{2}$ Department of Science and Technology, Linköping University, 60174 Norrköping, Sweden \\ (Received 2 September 2016; revised manuscript received 7 December 2016; published 3 February 2017)
}

\begin{abstract}
Ever since the first experimental investigations of organic field effect transistors (OFETs) the dimensionality of charge transport has alternately been described as two dimensional (2D) and three dimensional (3D). More recently, researchers have turned to an analytical analysis of the temperature-dependent transfer characteristics to classify the dimensionality as either $2 \mathrm{D}$ or $3 \mathrm{D}$ as well as to determine the disorder of the system, thereby greatly simplifying dimensionality investigations. We applied said analytical analysis to the experimental results of our OFETs comprising molecularly well-defined polymeric layers as the active material as well as to results obtained from kinetic Monte Carlo simulations and found that it was not able to correctly distinguish between 2D and 3D transports or give meaningful values for the disorder and should only be used for quasiquantitative and comparative analysis. We conclude to show that the dimensionality of charge transport in OFETs is a function of the interplay between transistor physics and morphology of the organic material.
\end{abstract}

DOI: 10.1103/PhysRevB.95.085301

\section{INTRODUCTION}

Understanding the charge transport mechanisms of organic field effect transistors (OFETs) is of great importance to the development of efficient devices serving a range of applications. Knowing where in a device charge transport occurs is particularly important for the development of thinfilm transistors as it determines the thickness limit of such devices, allowing to develop single monolayer devices that potentially are flexible, printable, and cheap to produce. To this end, the dimensionality of charge transport in OFETs has become a subject of discussion in recent years with a multitude of materials, such as quaterthiophene [1], sexithiophene (6T) [2-4], poly(3-hexylthiophene) (P3HT) [5], and naphthalenebis(dicarboximide)-dithiophene [P(NDI2OD-T2)] [6] having been subjected to studies. Typically, these studies of dimensionality include investigations of microstructure, transfer characteristics, and most notably the dependence of the mobility or drain current on the layer thickness, the latter usually being measured as the number of monolayers (MLs) that has been deposited. An early in situ study of the spatial carrier density distribution in pentacene and 6T showed an exponential decrease in the carrier density with distance from the dielectric/semiconductor interface independent of the applied gate field and an accumulation layer thickness of one to two and three to four MLs, respectively [7]. In studying the more recent literature a trend emerges where a saturation of the mobility within the first one to three MLs occurs followed by a thickness independence of the mobility at greater numbers of MLs if the layer deposition method allows for the film to develop a long-range ordered microstructure-often in the form of edge-on conformational order with $\pi-\pi$ stacking parallel to the substrate $[6,8]$. Such conditions typically are found in thermal evaporation at low rates as well as in solution-based Langmuir-Schäfer (LS) deposition [8,9]. In some systems, although not generally, thermal evaporation at high rates may lead to an increase in island formation and an overall less well-defined microstructure [9]. Spin coating generally does not allow for obtaining molecularly well-defined layers so that a study on the ML dependence is hampered [8]. Both lead to a ML dependence of the mobility that saturates within the first few (approximately three to six) MLs, depending on the material and specific growth parameters. An exception is presented in Ref. [10] where the asymmetric chain substitution of spin-coated NDI(2OD)(4tBuPh)-DTYM2 is believed to cause spontaneous long-range order of the microstructure resulting in a ML-independent mobility. It should be noted that thermal annealing of LS films results in the loss of the well-defined long-range order and the appearance of a ML dependence of the mobility [11]. The brief summary of previously published work given above serves to show that an experimental analysis of dimensionality is by no means straightforward and heavily influenced by experimental parameters, requiring not only electrical characterization, but also investigation of the microstructure, leading to varying interpretations of the dimensionality of the system in question. Additionally, definitions of what constitutes a ML may vary, and accurate control of the number of MLs is not always possible. As a consequence, one therefore often has to rely on indirect (analytical) methods to obtain the dimensionality of transport, which come with their own set of limitations. Furthermore, the question stands whether or not confinement of carriers occurs due to inherent properties of organic disordered semiconductors (ODSCs) or is the consequence of the gate field effect.

Despite the relatively large experimental body of work, the topic of dimensionality in OFETs has received very little attention from a theoretical point of view. A numerical investigation based on the Gaussian disorder model was successful in confirming the results in Ref. [7], i.e., a gate field-independent accumulation layer thickness that only spans across the first few MLs; however the effect of the spatial confinement on the carrier mobility in a thin film was not addressed [12]. A numerical analysis taking into account various thicknesses concluded that, whereas carriers are in fact confined to the first few MLs, charge transport in OFETs is generally a three-dimensional (3D) process [13]. However, in both cases an energetic disorder of the localized hopping states in the form of a Gaussian distribution is assumed, whereas commonly an exponential distribution is used in analyses of experiments. 
Early on, Vissenberg and Matters (VM) derived a general analytical expression for the field effect mobility in OFETs based on percolation theory and hopping in an exponential density of states (DOS) [14]. The VM model assumes a strong energy dependence of the occupation of available states and therefore only is valid in the tail of the DOS, i.e., at temperatures $T \leqslant T_{0}$, where $T_{0}$ is the characteristic temperature of the exponential DOS. Various researchers subsequently have employed the VM conductivity model to derive relationships between drain current and gate voltage $\left(V_{g}\right)$ that account for the differences between two-dimensional (2D) and 3D confinements. Like the original VM model, these "extended" VM models predict a power-law relationship between drain current and gate voltage $I_{D} \propto\left(V_{g}-V_{t}\right)^{\gamma}$, where $V_{t}$ is the threshold voltage. In the extended VM models (eVMs), the exponent $\gamma$ is different for 2D and 3D charge transports. In the linear transport regime the exponents follow to $\gamma^{2 D}=T_{0} / T$ and $\gamma^{3 D}=2 T_{0} / T-1$, respectively, where $T$ is the lattice temperature [15]. In the saturation regime the corresponding exponents follow to $\gamma^{2 D}=T_{0} / T+1$ and $\gamma^{3 D}=2 T_{0} / T$ for 2D and 3D transports, respectively [16].

The procedure to obtain the dimensionality of a system can, in short, be described as follows. From power-law fits to the transfer characteristics at different temperatures the exponent $\gamma$ is obtained and plotted against the inverse temperature. The intersection at $1 / T=0$ (the $\gamma$ axis) obtained from a linear fit to the $\gamma$ data indicates the dimensionality of the system and should only be one of two integer values to be conclusive. To show that this procedure is accurate in determining dimensionality, several material systems have previously been analyzed within the eVM framework. FETs operating in the linear regime with spin-coated layers of P3HT, poly(2,5-thienylene vinylene), and Poly[2-methoxy-5-( $3^{\prime}, 7^{\prime}-$ dimethyloctyloxy)-1,4-phenylenevinylene] of thicknesses far exceeding the channel thickness expected from electrostatics (several nanometers) were found to host 3D transport. Selfassembled monolayer field effect transistors with a single ML of dimethylsilane and FETs with an evaporated single ML of T6- where the spatial extension of the conduction channel is intrinsically limited to one ML-were found to be $2 \mathrm{D}$ in the linear regime [15]. Interestingly, dimensionality investigations according to the eVM of FETs operating in the saturation regime with bulk spin-coated layers of various polymers with thicknesses ranging from 60 to $100 \mathrm{~nm}$ showed 2D transport for all systems [16]. It should be mentioned that in Refs. [15,16] the linear fits of the temperature dependence of $\gamma$ were forced to fall on the best-matching integer value predicted by the eVM model.

Here we investigate experimentally and numerically the dimensionality of charge transport for organic systems commonly used in OFETs. On the basis of transfer characteristics measured on top-gate OFETs with various numbers of MLs of P(NDI2OD-T2) (also known as N2200) deposited in a layer-by-layer LS technique we show that, although the underlying physics of the VM model can be adapted to 2D transport by changing the critical number of bonds necessary for percolation, an analysis of dimensionality per the eVM was in fact incapable of characterizing 2D transport. Rather, we find that the analysis described above leads to a noninteger dimensionality that cannot be categorized exclusively as 2D or
3D. We find a remarkably similar conclusion when performing said dimensionality analysis on data obtained from kinetic Monte Carlo (MC) simulations that only account for the most basic physics of ODSCs. This numerical approach eliminates most problems of material- or deposition-method-dependent dimensionality and serves as a powerful tool to gain a more universal insight into charge transport in OFETs. Several realizations of the physical model representing a large group of organic semiconductors are investigated and interpreted leading to a clear picture in which the dimensionality of the transport is a consequence of the interplay between morphology and gate field. Furthermore, we show that an analysis of dimensionality according to the eVM model may, in some cases, lead to erroneous or unphysical conclusions for both dimensionality and disorder. It may, however, be used to identify qualitative differences. Additionally, we show that a Gaussian DOS as used in previously published numerical works cannot be used to describe the charge transport in OFETs in our case as it leads to a virtual absence of a $V_{g}$ dependence of the mobility.

\section{EXPERIMENTS}

The experimental basis of our paper is formed by the electrical characterization of top-gate OFETs with various numbers of P(NDI2OD-T2) MLs. The fabrication and deposition protocol of P(NDI2OD-T2) MLs has been described in detail in a number of previous publications [6,8]. In brief, $\mathrm{P}(\mathrm{NDI} 2 \mathrm{OD}-\mathrm{T} 2)$ is self-organized by means of LS in molecularly well-defined $\sim 3$-nm-thick layers. Each layer is a close-packed monolayer having a precise out-of-plane molecular order. The individual P(NDI2OD-T2) monolayers are then deposited in a layer-by-layer fashion onto a glass substrate with prepatterned source/drain electrodes. See the Methods section for further details on the fabrication process.

In Fig. 1(a) the transfer characteristics at room temperature of OFETs with one to three and five MLs are shown in the form of the gate voltage dependence of the mobility calculated from the measured drain currents that are shown in the inset of Fig. 1(a). The data were obtained in the linear operation regime at a drain-source voltage $V_{\mathrm{DS}}=5 \mathrm{~V}$. Contrary to most previously reported devices, one can see that throughout the gate voltage range there is very little to no difference in mobility between OFETs with channels of one to three MLs. The small decrease in the mobility can be attributed to an increasing loss of the well-defined LS microstructure by the stacking of the individual layers. This effect continues with every added LS layer and results in a somewhat stronger decreased mobility of the five-ML device. Importantly, these observations give direct evidence that there is no significant gain in mobility by adding additional material to the channel. In the saturation regime at $V_{\mathrm{DS}}=70 \mathrm{~V}$ the previously described small decrease in mobility for thicker devices vanishes at higher gate fields, whereas a clear hysteresis arises at lower gate voltages as well as a ML-dependent threshold shift (see Fig. S3 in the Supplemental Material [17]). For this reason, we will focus this paper on the-also more physically transparent-linear regime.

In order to analyze the dimensionality according to the process described above, transfer characteristics for all devices 


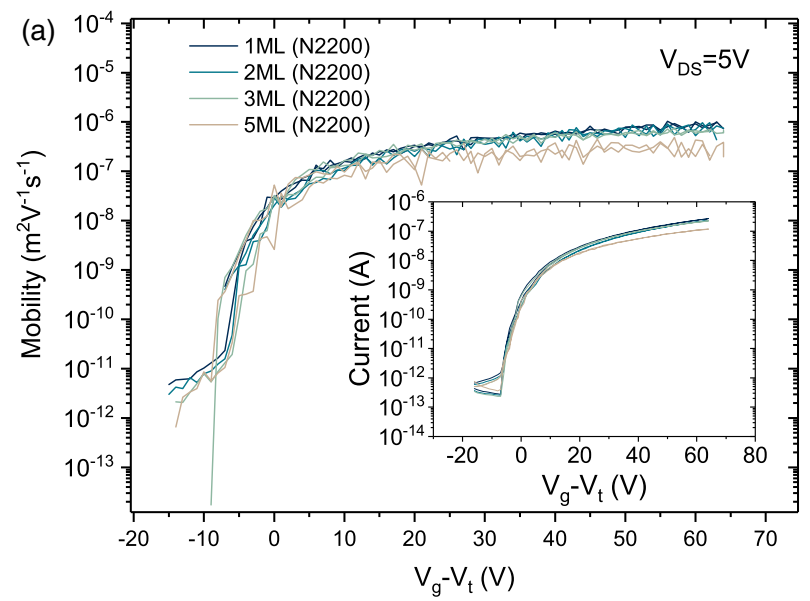

(b)

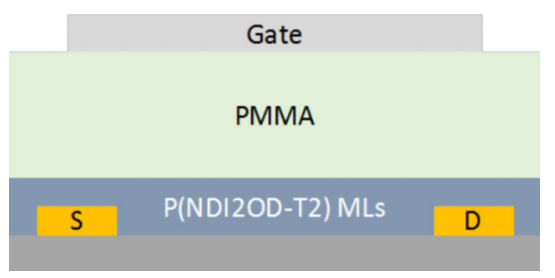

FIG. 1. (a) Linear mobility of monolayer OFETs plotted as a function of gate voltage. The inset shows the gate field dependence of the current from which the mobility has been calculated. (b) Schematic of the top-gate OFETs used in this paper.

were recorded at various temperatures. As an example, in Fig. 2(a) the transfer characteristics for a two-ML OFET in the linear regime are plotted on a double-logarithmic scale parametric in temperature ranging from 180 to $300 \mathrm{~K}$. The transfer characteristics for other thicknesses are plotted in Fig. S1 of the Supplemental Material [17]. The solid lines are power-law fits to the linear part of the transfer curves (on the double-logarithmic scale) found at high gate voltages. One can clearly see that the power-law relation between drain current and gate voltage necessary for the eVM analysis only holds at high gate voltages (which also constitutes the operation regime of a FET) with deviations at low voltages.

The $\gamma$ values for all devices at each temperature are extracted from the inclination of these fits and are shown in Fig. 2(b) plotted against the inverse temperature $1 / T$. We did not enforce an intersection with the $\gamma$ axis at any of the values predicted by the eVM model but rather applied unbiased linear fitting to obtain the intersections. The data obtained that way show no clear correlation between the thickness of the channel and the dimensionality obtained from this analysis as can be seen in the inset of Fig. 2(b). In fact, all intersections would indicate a sub-2D transport regime indicating that the experimental data cannot be described by the extended VM model. We performed the same procedure of power-law fittings at lower gate voltages and found that although $\gamma$ remained largely the same, the intersections shift down to approximately one (with the exception of five MLs), i.e., still indicating sub-2D transport. The corresponding plots can be found in Figs. S2(a) and S2(b) of the Supplemental Material [17].

\section{SIMULATIONS}

To gain a deeper understanding of the physical processes that determine the dimensionality in the active material of OFETs we have performed MC simulations modeled on the devices used in our experimental investigations. Our numerical model consists of a gate contact, dielectric material, semiconductor material, and a substrate, which are stacked on top of each other along the $z$ axis. A gate field is applied in the $-z$ direction populating the semiconductor with holes. Perpendicular to the gate field a smaller field is applied determining the direction of current flow. No source or drain contacts are present. Details on the MC algorithm are described in the Methods section below and in Ref. [18]. In short, we consider a box containing localized sites distributed on a simple cubic lattice in space and according to an exponential or Gaussian distribution in energy. The unit cell of the lattice was chosen to be rectangular with the dimensions of $1 \times 1 \times 3 \mathrm{~nm}^{3}$, mimicking the structure of LS deposited N2200 reported in Ref. [6]. For comparison we performed the same set of simulations on a lattice with a cubic unit cell of size $1 \times 1 \times 1 \mathrm{~nm}^{3}$. Charge transport of carriers occurs via nearest-neighbor hopping $(\mathrm{NNH})$ between these sites with rates given by the Miller-Abrahams expression for hopping transport. We justify the choice of $\mathrm{NNH}$ over variable range hopping by the relatively high charge-carrier concentrations in our simulated devices as well as the fact that the probability for a hop in a strongly energy-dependent DOS, such as an exponential or a Gaussian, is more sensitive to energy than to distance. The hopping probabilities in the $x, y$, and $z$ directions scale exponentially with the lattice constant of the unit cell in the respective direction as described in the Methods section. Furthermore, our numerical simulations include Coulomb interactions between all particles, their twin charges due to periodic boundary conditions up to five box sizes away, as well as all corresponding image charges in the case of a dielectric contrast between semiconductor and substrate. All input parameters for the simulation have been chosen to physically be sound and close to what one would expect for the common systems investigated experimentally here and in previously published literature.

In the first step of the numerical investigation we made sure that our simulations satisfyingly reproduce the transfer characteristics described above. In Fig. 3(a) the experimental data from Fig. 1(a) are compared to results of the MC simulations of a rectangular lattice. As there is (by design) no threshold voltage for the simulated devices, comparison with the experimental data revealed that our N2200 OFETs have a threshold voltage of $V_{t}=6 \mathrm{~V}$. We therefore have corrected all experimental data by plotting them as a function of $V_{g}-V_{t}$. We found that a disorder of $50 \mathrm{meV}$ best reproduces the gate field dependence of the mobility observed in the experiments. 


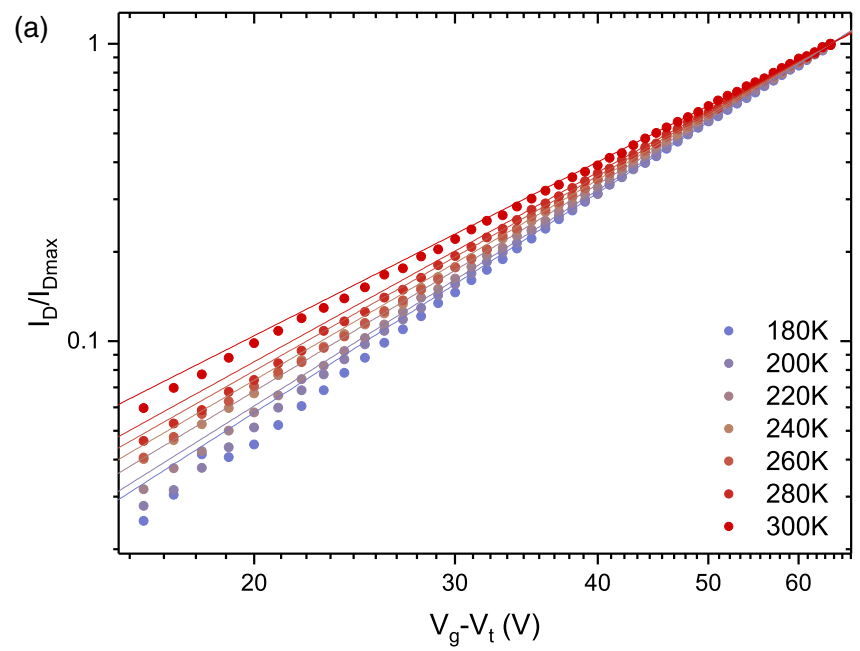

(b)

FIG. 2. (a) Transfer characteristics of a two-ML P(NDI2OD-T2) OFET at different temperatures on a double-logarithmic scale. The symbols are experimentally obtained data points, and the solid lines are power-law fits to the high gate voltage range of these data. (b) $\gamma$ values obtained from the inclination of the power-law fits to the transfer characteristics (symbols) vs inverse temperature. The lines are linear fits extrapolated to the $\gamma$ axis. The intersections should indicate the dimensionality of the system. The inset shows said intersections as a function of the number of MLs.

The MC simulations on a cubic lattice, shown in Fig. S4 of the Supplemental Material [17], exhibited a different gate field dependence and in general show much less agreement with the experiments, especially at lower fields. The hopping attempt frequency was set (at $\sim 10^{12} \mathrm{~Hz}$ ) to match the magnitude of the experimental mobility. As described above, the mobility decreases slightly with increasing number of MLs in the experiments due to morphological defects. However, in the simulations no such effects exist, and instead we observe a small increase in mobility with thicker layers. The increase in mobility is due to the fact that with more MLs there are more hopping sites available in the $z$ direction for the charge carriers to avoid difficult in-plane hops.

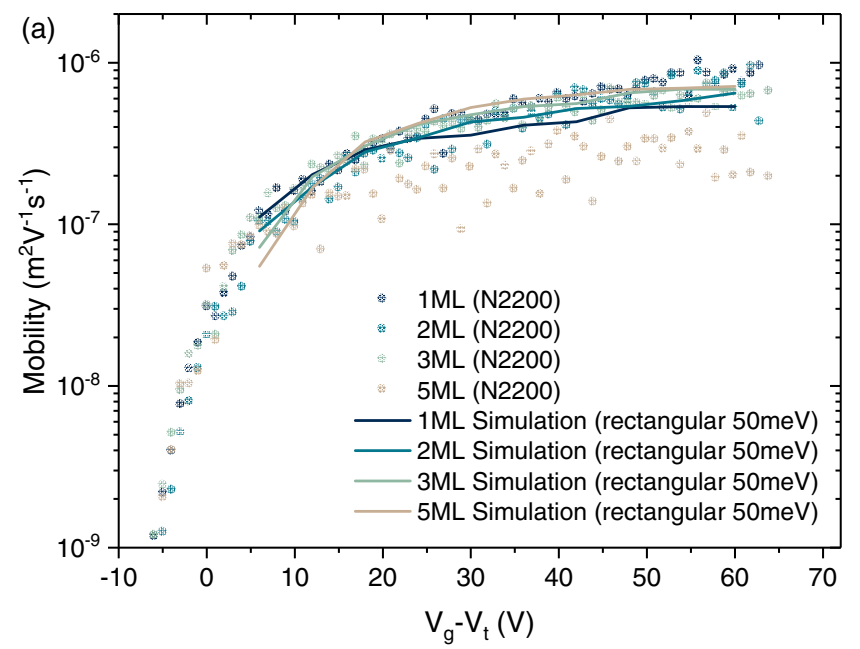

In our simulations, the charge transport in the energetically disordered channel of OFETs is determined by three factors; Coulomb interaction (i.e., scattering) between carriers, statefilling, and (the degree of) confinement of the carriers to the semiconductor/dielectric interface, i.e., the dimensionality. The interplay between these factors has been discussed extensively in Ref. [13] and will therefore, in the context of the present paper, only shortly be addressed in the following.

In Fig. 3(b) we studied the disorder and gate field dependence of the transfer characteristics with MC for a rectangular system with various magnitudes of exponential disorder, each with various numbers of MLs. Similar data for a cubic lattice are shown in Fig. S5(a) of the Supplemental Material [17]. As mentioned earlier, in previously published

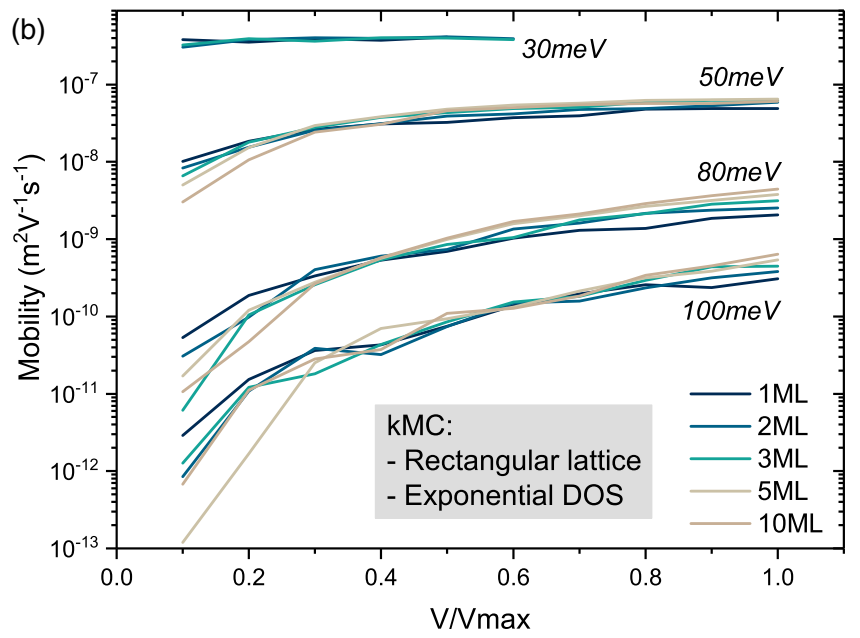

FIG. 3. (a) The solid lines are the calculated mobilities as a function of gate field for simulated devices with a rectangular lattice and thicknesses ranging from one to five MLs, which are compared to experimental curves of P(NDI2OD-T2) OFETs from Fig. 1(a) (full symbols). (b) Comparison of the mobilities calculated for a rectangular lattice with exponential energetic disorder for various numbers of MLs as indicated by the color of the lines and disorders of $30,50,80$, and $100 \mathrm{meV}$. 

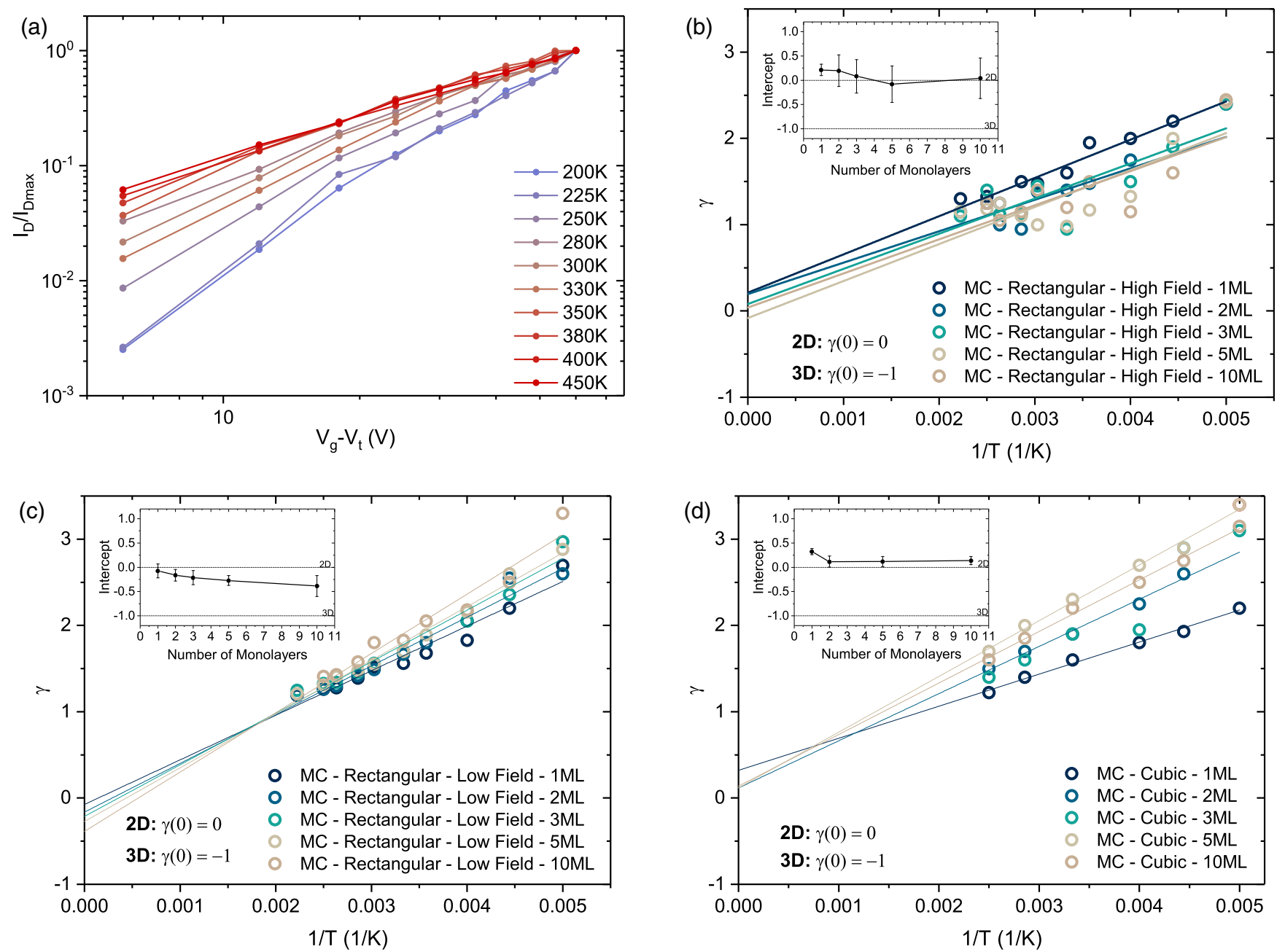

FIG. 4. (a) Simulated transfer characteristics at different temperatures of OFETs with two MLs using a rectangular lattice for hopping (dots). The solid lines are guides to the eye. (b)-(d) $\gamma$ values plotted against inverse temperature (open circles) and linear fits extrapolated to the $\gamma$ axis (solid lines) for (b) a rectangular lattice at high fields, (c) a rectangular lattice at low fields, and (d) a cubic lattice for the whole field range. The insets in (b)-(d) show the intercept with the $\gamma$ axis as a function of the number of MLs. The dashed lines indicate intercepts for $2 \mathrm{D}$ and $3 \mathrm{D}$ according to eVM.

numerical investigations of OFETs a Gaussian distribution was assumed for the DOS $[12,13]$. Although this assumption might be correct for ODSCs in general, it is not commonly used for analyzing experimental investigations of OFETs [14-16,19]. In fact we found that regardless of the value for the width of the DOS, a Gaussian distribution leads to a near-constant mobility over the whole field range and, in fact, slightly decreases with increasing gate field. The difference between an exponential and a Gaussian DOS can clearly be seen in Fig. S5(b) of the Supplemental Material [17]. This has been observed before and attributed to an increased effect of state filling at higher disorders [13]. However, a Gaussian DOS does not reproduce our experimental findings and was therefore discarded for the remainder of this paper.

Using an exponential DOS, we observe an increase in gate field dependence with increasing disorder, which is explained by the well-known effects of state filling that become increasingly important at higher disorders. The changing field dependence with the characteristic temperature $T_{0}$ of the exponential DOS allows us to estimate the disorder of the material used in the experiments of this paper to be around $50 \mathrm{meV}$. Additionally, upon close inspection of the low-field regime of the simulated curves in Fig. 3(a) it can be seen that the ML dependence of the mobility inverts and a decrease (increase) in the mobility with increasing thickness occurs for high (low) disorder. This confirms the finding in Ref. [13] that confinement effects can have either a positive or a negative effect on the mobility, depending on disorder. For high disorder, the positive effects of state filling that are associated with an increased confinement (thinner active layer or higher gate field) dominate over the negative effects of enhanced Coulomb scattering and a reduced number of pathways in the $z$ direction. At low disorder, state filling is less important, and the effect of increased confinement inverts. This effect is more pronounced in a cubic lattice where the charges spread out more easily over multiple layers.

We continue by analyzing the MC data in Fig. 3(a) according to the extended VM model in the same manner as the experiments. The current scaled to its value at the highest gate voltage is plotted against gate voltage in Fig. 4(a) on a double- 

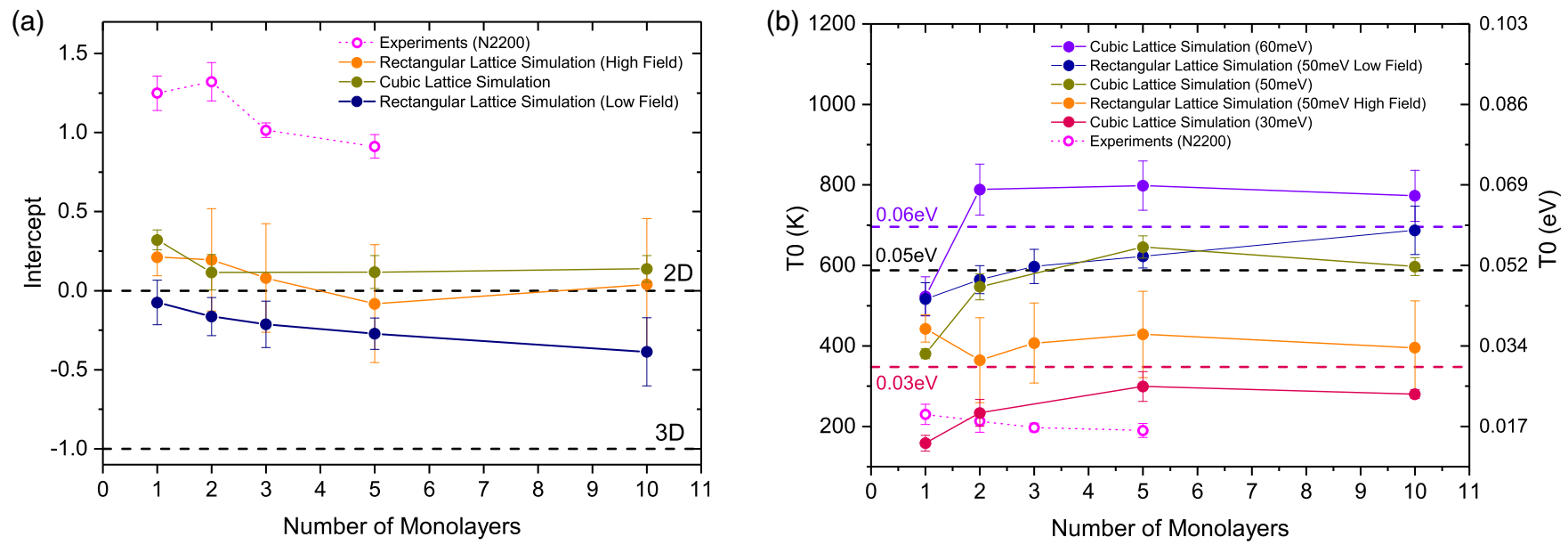

FIG. 5. (a) Intercepts with the $\gamma$ axis for all cases discussed above as a function of the number of MLs. The dashed line corresponds to 2D and $3 \mathrm{D}$ behaviors according to the eVM model. (b) Extracted width $T_{0}$ of the exponential DOS. The dashed line indicates the input $T_{0}$ of the MC simulations.

logarithmic scale for a two-ML device on a rectangular lattice at temperatures ranging from 200 to $450 \mathrm{~K}$. The equivalent plots for other thicknesses as well as those for devices with a cubic lattice can be found in Figs. S7 and S6 of the Supplemental Material [17], respectively. Fitting power laws to the transfer curves obtained for a rectangular lattice presented a problem in that they do not follow a power law over the full gate voltage range considered-note that a similar problem was encountered in the experimental data [Fig. 2(a)]. Rather, there appear to be two power laws with different exponents. Furthermore, at high fields all curves seem to fall on the same line with the exception of the lowest two temperatures. This indicates that thermal activation of carriers only plays a minor role above a certain gate voltage range. The reason for this can be found in the fact that at high gate fields the carriers are already close to the transport energy and require only little thermal activation for hopping. Continuing the next step of the analysis, we extract $\gamma$ values from power-law fits to the high-field regime (second half of the gate voltage range) and plot the data against the inverse temperature in Fig. 4(b). The large scatter in the data points indicates that the extrapolated interceptions should be considered as being merely indicative. Nevertheless, the resulting intersections with the $\gamma$ axis [insets in Figs. 4(b) and 5(a)] would suggest sub-2D to 2D transport depending on the number of MLs, in reasonable agreement with the experiment in Fig. 2(b). Two-dimensional transport is what one would intuitively expect for carriers that are confined by both a rectangular lattice and a high gate field.

The $\gamma$ values extracted from the low-field regime (first half of the gate voltage range) are plotted against the inverse temperature in Fig. 4(c). As can be seen a reasonable linearity is observed, and the obtained intersections range from approximately 0 to -0.5 with smaller error bars, seeming to indicate a $2 \mathrm{D}$ to $3 \mathrm{D}$ transition with the system getting more 3D with an increasing number of MLs. In principle, this observation would be consistent with a smaller gate field leading to less confinement and a concomitant higher degree of three dimensionality. Below we will show that this is merely a spurious correlation.
The same analysis performed on MC data where hopping occurs on a cubic lattice is more straightforward. The transfer curves largely follow a power law, and the extracted $\gamma$ data are linear in temperature as can be seen in Fig. 4(d). The fact that the currents are analyzable in the cubic case could be ascribed to the more 3D-like nature of the cubic lattice, bringing the system closer to the assumptions underlying the VM transport model. However, similar to the experiments and the rectangular lattice, the intersections obtained suggest an unphysical sub-2D transport, especially for the one-ML case.

According to the extended VM model the slopes of the linear fits in Figs. 2(b) and 4 give the width $T_{0}$ of the exponential DOS, which is plotted in Fig. 5(b) for all cases discussed above. For two or more MLs of the cubic lattice and the rectangular lattice analyzed at low fields the input $T_{0}$ of $\sim 600 \mathrm{~K}(50 \mathrm{meV})$ is recovered in the analysis. The values obtained for the rectangular lattice analyzed at high fields are around $400 \mathrm{~K}$, whereas those extracted from the experiments all fall below $300 \mathrm{~K}$. It is important to note that the original VM model is only valid at temperatures $T<T_{0}$, meaning that a posteriori the analysis of the experiments is largely invalid by this criterion. Further analysis of MC data for a cubic lattice with input disorders of 30 and $60 \mathrm{meV}$ is plotted in Fig. 5(b) and shows that, although the eVM analysis underestimates $T_{0}$ at low disorders, it increasingly overestimates $T_{0}$ at higher input disorders.

Summarizing our results so far, we have shown that the eVM analysis with a free intercept of the experimental system investigated in this paper results in nonphysical dimensionalities $(<2)$ and internally inconsistent $T_{0}$ values. Conclusions drawn from an analysis of MC simulation data of a system where hopping occurs on a (realistic) rectangular lattice, thus favoring $2 \mathrm{D}$ transport, depended on the field range. If performed in the high-field regime as suggested in Ref. [15], the linearity of $\gamma$ in temperature is lost to a large degree, and an analysis becomes cumbersome. Analysis of the rectangular lattice in the low-field regime, however, is possible and seems to point towards a 2D to $3 \mathrm{D}$ transition. However, simulations on a cubic lattice on which a $2 \mathrm{D}$ to $3 \mathrm{D}$ transition is more likely 

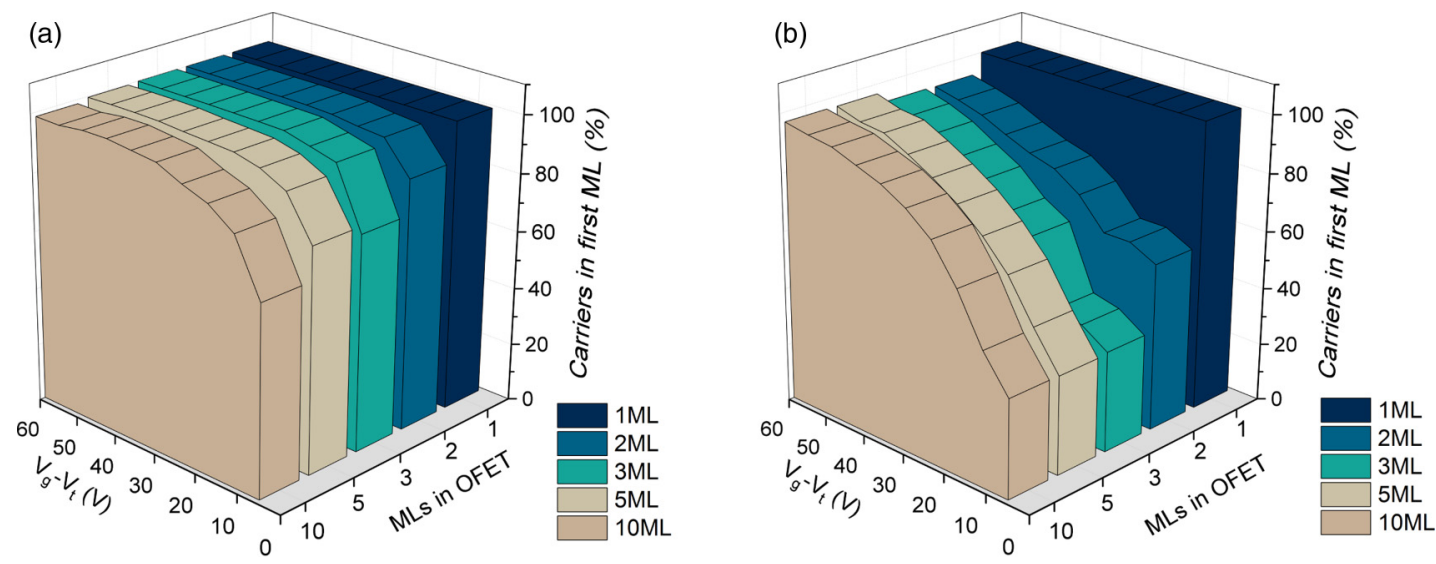

FIG. 6. Percentage of carriers in the first ML as a function of gate voltage and total number of MLs in the device for (a) a rectangular lattice and (b) a cubic lattice.

showed no indication of such a transition, despite the fact that the cubic system could be better analyzed with the eVM model. It is noteworthy that the eVM analysis did not point to a clear 2D behavior for transport in one ML for any of the MC systems investigated here. Moreover, the disorder parameters extracted from the eVM analysis can show significant deviations from the true value.

Kinetic MC allows us to follow charge carriers over time and determine their position in the device once convergence of the simulation has been reached. This creates the unique possibility to unambiguously determine whether charges are distributed over the whole device or confined to a single layer, i.e., whether the charge-carrier distribution is $2 \mathrm{D}$ or 3D. To this end we plotted the percentage of carriers in the first ML as a function of gate field and the number of MLs in the device for a rectangular and a cubic lattice in Figs. 6(a) and $6(\mathrm{~b})$, respectively.

As can be seen, in the case of a rectangular lattice most carriers are confined to the first ML already at low gate fields and are fully confined to the first ML in the high gate field range where the difference between the various devices becomes negligible. Even for the ten-ML device, more than $60 \%$ of the carriers are confined within the first ML already at the lowest gate field, and the confinement steeply increases thereafter. This suggests that transport in a rectangular lattice is largely 2D regardless of the number of MLs or gate field. Interestingly, this conclusion is somewhat similar to the results of the eVM analysis of the rectangular lattice in Fig. 5(a) if one is willing to ignore the large error bars and the absence of linearity in Fig. 4(a). In the case of a cubic lattice in Fig. 6(b) we see that at the low gate field only around $20 \%$ of the carriers are confined in the first ML for thicker devices and that the population gradually increases until the gate field confines most carriers to the first ML. This clearly indicates a transition from 3D to 2D with increasing gate field. However, the analysis in Fig. 5(a) points to sub-2D transport for all thicknesses, which is in clear contrast to the actual distribution of carriers in Fig. 6(b).

\section{CONCLUSION}

In conclusion, we have investigated the transfer characteristics of LS deposited N2200 OFETs and analyzed the dimen- sionality of the charge transport by means of the extended Vissenberg-Matters model. We found that this model cannot be used to make absolute statements about the dimensionality of charge transport and that enforcing an extrapolation of the fitted power-law exponents $\gamma$ through the nearest integer is prone to lead to misleading identification of 2D transport. For further analysis we performed MC simulations of OFETs modeled on the $\mathrm{N} 2200$ devices using a rectangular or a cubic lattice and exponential or Gaussian disorder. We find that Gaussian disorder does not reproduce the gate field dependence of the mobility from experiments, whereas an exponential disorder does. Extended VM analysis of the MC data results in the same conclusion as for the experiments, namely, that this analysis cannot properly identify $2 \mathrm{D}$ or $3 \mathrm{D}$ transport. This is further supported by an analysis of the spatial charge distribution of the simulated OFETs. In addition, the extended VM analysis was found to be ill suited for quantitative determination of the disorder $T_{0}$.

\section{METHODS}

\section{A. Device fabrication and characterization}

P(NDI2OD-T2) was purchased from Polyera Corp. (ActivInk $\left.^{\mathrm{TM}} \mathrm{N} 2200\right)$ and used as received. Monolayers and multilayers of P(NDI2OD-T2) were prepared by the LS technique following the procedure described in Ref. [6]. A $0.2-\mathrm{mg} / \mathrm{ml}$ chloroform solution of P(NDI2OD-T2) was spread onto the water subphase. After solvent evaporation, the floating film was compressed in order to induce a compact packing of the molecules at the air/water interface. The deposition was then carried out at a surface pressure of about $25 \mathrm{mN} / \mathrm{m}$ by approaching hexamethyldisilazane-treated glass substrates horizontally to the water subphase. This surface pressure allows for the self-assembly of well-ordered films with a preferential edge-on molecular orientation with respect to the substrate over a large area [6].

Ordered monolayers and multilayers of P(NDI2OD-T2) were deposited on Corning glass substrates with photolithographically patterned source/drain electrodes (3-nm-thick Ti and 12-nm-thick $\mathrm{Au}, L=20 \mu \mathrm{m} / \mathrm{W}=1000 \mu \mathrm{m})$. The films were then annealed at $120^{\circ} \mathrm{C}$ in vacuum overnight. Next, polymethylmethacrylate (PMMA) $(M w=120 \mathrm{kDa}$, Sigma- 
Aldrich) was dissolved in 2-butanone (MEK) at a concentration of $70 \mathrm{mg} \mathrm{mL}^{-1}$, spin coated on top of the semiconductor thin layer, and annealed at $120^{\circ} \mathrm{C}$ in vacuum for $3 \mathrm{~h}$. Finally, an 80-nm-thick Al gate electrode was thermally evaporated on top of the dielectric layer. Electrical measurements were performed in vacuum by using a cryogenic probe station (Janis Research Company) connected to a Keithley 4200-SCS semiconductor parameter analyzer.

\section{B. Kinetic Monte Carlo simulation}

The MC experiments were performed at different temperatures in a box containing $70 \times 70 \times N$ lattice sites in the $x, y$, and $z$ directions, respectively, where $N$ is the number of monolayers. As outlined above, the intersite distance of the rectangular hopping lattice was chosen to be $1 \times 1 \times 3$ and $1 \times 1 \times 1 \mathrm{~nm}^{3}$ for cubic lattices. The hopping probability $p_{d}$ is modified with distance by $p_{d} \propto e^{-2 \alpha\left(a_{d}-a_{0}\right)}$, wherein $\alpha=2 \times 10^{9} \mathrm{~m}^{-1}$ is the inverse localization length, $a_{d}$ is the lattice constant in the direction of the hop, and $a_{0}$ is a reference lattice constant. The number of particles in the box is set by $V_{g}$ and the areal capacitance of the gate and was between several tens and several hundreds for the field range considered in this paper. Every data point is obtained by averaging over
20 configurations where convergence of every simulation was assured. Interactions of twin charges resulting from the periodic boundary conditions in the $x$ and $y$ directions have been included for all carriers up to five box sizes away at which point these interactions become negligible. Image charges of all charges and their twins in the $z$ direction arise due to the difference in dielectric constant between the semiconductor $\epsilon_{1}=3.6$ and the substrate $\epsilon_{2}=4.7$ (the gate dielectric was set to have the same dielectric constant as the semiconductor). The electric potential in the semiconductor due to self-interaction of a charge $q$ was calculated according to Ref. [13] as $\frac{q_{t}}{4 \pi \epsilon_{0} r}$, where $\epsilon_{0}$ is the vacuum permittivity, $r$ is the distance between the charge and its image in the substrate in the $z$ direction, and $q_{t}=\frac{q}{\epsilon_{1}}\left(\frac{\epsilon_{1}-\epsilon_{2}}{\epsilon_{1}+\epsilon_{2}}\right)$ is the total charge induced by a charge $q$ in the substrate.

\section{ACKNOWLEDGMENTS}

H.A. gratefully acknowledges funding by the Knut och Alice Wallenbergs stiftelse (project 'Tail of the Sun'). S.F. acknowledges support from the Advanced Functional Materials Center at Linköping University (Grant No. 2009-00971) and VINNOVA (Grant No. 2015-04859).
[1] T. Muck, V. Wagner, U. Bass, M. Leufgen, J. Geurts, and L. W. Molenkamp, Synth. Met. 146, 317 (2004).

[2] A. Dodabalapur, L. Torsi, and H. E. Katz, Science 268, 270 (1995).

[3] E. L. Granstrom and C. D. Frisbie, J. Phys. Chem. B 103, 8842 (1999).

[4] F. Dinelli, M. Murgia, P. Levy, M. Cavallini, F. Biscarini, and D. M. de Leeuw, Phys. Rev. Lett. 92, 116802 (2004).

[5] H. G. O. Sandberg, G. L. Frey, M. N. Shkunov, H. Sirringhaus, R. H. Friend, M. M. Nielsen, and C. Kumpf, Langmuir 18, 10176 (2002).

[6] S. Fabiano, C. Musumeci, Z. Chen, A. Scandurra, H. Wang, Y.-L. Loo, A. Facchetti, and B. Pignataro, Adv. Mater. 24, 951 (2012).

[7] M. Kiguchi, M. Nakayama, T. Shimada, and K. Saiki, Phys. Rev. B 71, 035332 (2005).

[8] S. Fabiano, H. Yoshida, Z. Chen, A. Facchetti, and M. A. Loi, ACS Appl. Mater. Interfaces 5, 4417 (2013).

[9] R. Ruiz, A. Papadimitratos, A. C. Mayer, and G. G. Malliaras, Adv. Mater. 17, 1795 (2005).

[10] F. Zhang, C. Di, N. Berdunov, Y. Hu, Y. Hu, X. Gao, Q. Meng, H. Sirringhaus, and D. Zhu, Adv. Mater. 25, 1401 (2013).
[11] V. D’Innocenzo, A. Luzio, H. Abdalla, S. Fabiano, M. A. Loi, D. Natali, A. Petrozza, M. Kemerink, and M. Caironi, J. Mater. Chem. C 4, 11135 (2016).

[12] L. Demeyu, S. Stafström, and M. Bekele, Phys. Rev. B 76 , 155202 (2007).

[13] A. Sharma, F. W. A. van Oost, M. Kemerink, and P. A. Bobbert, Phys. Rev. B 85, 235302 (2012).

[14] M. C. J. M. Vissenberg and M. Matters, Phys. Rev. B 57, 12964 (1998).

[15] J. J. Brondijk, W. S. C. Roelofs, S. G. J. Mathijssen, A. Shehu, T. Cramer, F. Biscarini, P. W. M. Blom, and D. M. de Leeuw, Phys. Rev. Lett. 109, 056601 (2012).

[16] A. J. Kronemeijer, V. Pecunia, D. Venkateshvaran, M. Nikolka, A. Sadhanala, J. Moriarty, M. Szumilo, and H. Sirringhaus, Adv. Mater. 26, 728 (2014).

[17] See Supplemental Material at http://link.aps.org/supplemental/ 10.1103/PhysRevB.95.085301 for additional experimental and numerical plots concerning the material in the main text.

[18] A. Melianas, V. Pranculis, A. Devižis, V. Gulbinas, O. Inganäs, and M. Kemerink, Adv. Funct. Mater. 24, 4507 (2014).

[19] A. F. Basile, A. Kyndiah, F. Biscarini, and B. Fraboni, J. Appl. Phys. 115, 244505 (2014). 